\section{Gen-Expressions-Signatur bei Kolonkarzinom im Stadium II Neues Tool zur Prognoseabschätzung}

Für Patienten mit einem Kolonkarzinom (CC) im Stadium II und hohem Risiko wird eine adjuvante Chemotherapie empfohlen. Doch mehrere Studien weisen darauf hin, dass die bisher verwendeten histologischen und klinischen Marker nur eine eingeschränkte prognostische Aussagekraft aufweisen. Neue Daten zeigen nun, dass der ColDx-Assay bei Patienten mit CC im Stadium II ohne Hochrisikofaktoren bei Vorliegen eines Defekts in der Mismatch-Reparatur (MMR) mit der rezidivfreien Zeit assoziiert ist. Damit könnte dieser Test zusammen mit den traditionellen klinischen Risikomarkern verwendet werden, um Aussagen zur Prognose der Patienten zu präzisieren.

Für die aktuelle Analyse wurden die in Pafafin eingebetteten Gewebeproben verwendet, die den Patienten im Rahmen der Phase-III-Studie C9581 der Alliance for
Clinical Trials in Oncology (Alliance; ehemals Cancer and Leukemia Group B, CALGB) entnommen wurden. In der C9581-Studie war die Wirksamkeit von Edrecolomab im Vergleich zur alleinigen Beobachtung an 1.738 CC-Patienten im Stadium II ohne Hochrisikofaktoren untersucht worden [Niedzwiecki D et al. J Clin Oncol. 2011;29(23):3146-52]. Die Proben von 393 Patienten (360 Patienten aus der randomisiert ausgewählten Subkohorte der Studie C9581 und 33 weitere Patienten) wurden mit dem ColDx-Assay analysiert. Die Patienten wurden anhand der ColDx-Assay-Ergebnisse in Gruppen mit hohem bzw. niedrigem Risiko eingeteilt. 55\% der Patienten wurden als Hochrisikopatienten klassifiziert.

Nach der Adjustierung für prognostische Faktoren wie dem MMR-Defekt hatten Patienten, die gemäß ColDx ein ho-

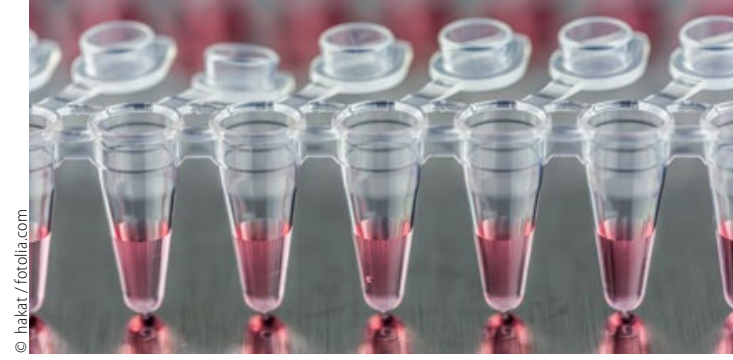

Gen-Expressions-Signatur: Entscheidungshilfe für oder gegen eine Chemotherapie.

hes Risiko aufwiesen, eine signifikant kürzere rezidivfreie Zeit (Hazard Ratio 2,03, $\mathrm{p}<0,01)$. Nach fünf Jahren betrug die Wahrscheinlichkeit für Rezidivfreiheit bei Hochrisikopatienten $82 \%$ im Vergleich zu $91 \%$ bei Patienten mit niedrigem Risiko. Beim Gesamtüberleben bestand kein signifikanter Zusammenhang zum ColDx-Array-Ergebnis (HR 1,74; p $=0,06$ ).

Judith Neumaier

Niedzwiecki D et al. Association Between Results of a Gene Expression Signature Assay and Recurrence-Free Interval in Patients With Stage II Colon Cancer in Cancer and Leukemia Group B 9581 (Alliance). J Clin Oncol. 2016;34(25):3047-53.

\section{kurz notiert}

Multiples Myelom:

Serum-FLC- schlägt Urinanalyse

Mit den Serumkonzentrationen der freien Leichtketten (sFLC) gelingt ein sensitiverer Nachweis des Leichtketten-Myeloms (LCMM) als mit Urinanalysen des monoklonalen Proteins. Jetzt gibt es erstmals Belege dafür, dass auch das Therapieansprechen und die Prognose durch sFLC-Messungen besser abgebildet werden. Thomas Dejoie und Kollegen haben bei 113 LCMM-Patienten, die an einer randomisierten Therapiestudie teilgenommen hatten, die Aussagekraft von Serumbestimmungen der involvierten (= tumorassoziierten) Leichtkette (iFLC) mit der Urinprotein-Elektrophorese verglichen [Dejoie T et al. Blood. 2016;Oct 11 (Epub ahead of print)]. Dass die Serum-FLC-Messungen tatsächlich die Krankheit besser widerspiegelten (und die Urinmessungen das Therapieansprechen überschätzten), zeigte sich beim progressionsfreien Überleben: Patienten, die nach dem dritten Zyklus einen erhöhten iFLCWert oder ein abnormes Verhältnis von Kappazu Lamda-Leichtketten im Serum aufwiesen, hatten ein signifikant kürzeres progressionsfreies Überleben als Patienten mit unauffälligen Werten. Die Befunde bei der Urinprotein-Elektrophorese oder Immunfixation im Urin standen dagegen nicht in Beziehung zum progressionsfreien Überleben. Beate Schumacher

Chronische myeloische Leukämie

\title{
TKI der zweiten Generation wirksamer
}

Die finalen Ergebnisse der Phase-III-Studie DASISION unterstützen den Einsatz von Dasatinib als effiziente und sichere Erstlinientherapie in der Langzeitbehandlung der chronischen myeloischen Leukämie (CML) in der chronischen Phase, auch wenn sich die beiden Therapiegruppen im progressionsfreien Überleben und Gesamtüberleben am Ende der Studie nicht unterschieden. In der Studie hatten Patienten mit neu diagnostizierter CML in der chronischen Phase entweder den Tyrosinkinasehemmer der zweiten Generation Dasatinib oder Imatinib erhalten. Traten erhebliche Nebenwirkungen auf, wurde die Therapie unterbrochen oder die Dosis reduziert. Bei suboptimalem Ansprechen nach 3 bis 18 Monaten war eine Anhebung der Dosis erlaubt.

Die kumulativen 5-Jahres-Raten des guten molekularen Ansprechens (MMR) betrugen 76 vs. $64 \%$ ( $p=0,0022)$ und des molekularen Ansprechens (MR) 42 vs. $33 \%$ unter Imatinib ( $\mathrm{p}=0,0251)$. Die Rate für das 5-Jahres-Gesamtüberleben be- trug $91 \%$ unter Dasatinib und $90 \%$ unter Imatinib. Auch die Rate für das progressionsfreie 5-Jahres-Überleben war in beiden Gruppen vergleichbar ( 85 vs. $86 \%$ ).

Patienten, bei denen BCR-ABL1 nach drei Monaten auf $\leq 10 \%$ gesunken war (84\% unter Dasatinib, 64\% unter Imatinib), erreichten in den fünf Jahren häufiger ein vollständiges zytogenetisches Ansprechen (cCCyR), ein MMR und $\mathrm{MR}^{4,5}$, hatten ein besseres progressionsfreies Überleben und ein besseres Gesamtüberleben als Patienten mit BCR-ABL1 > $10 \%$. Zudem ging bei ihnen die Krankheit seltener in die akzelerierte/Blastenphase über (3 vs. 14-15\%). Insgesamt geschah die Transformation in die akzelerierte/ Blastenphase bei $5 \%$ der Dasatinib-Patienten und $7 \%$ der Imatinib-Patienten.

Kathrin von Kieseritzky

Cortes J E et al. Final 5-Year Study Results of DASISION: The Dasatinib Versus Imatinib Study in Treatment-Naive Chronic Myeloid Leukemia Patients Trial. J Clin Oncol. 2016;34(20):2333-40. 